\title{
Simultaneous sensing and actuation with a piezoelectric tube scanner
}

\author{
S. O. Reza Moheimani and Yuen K. Yong \\ School of Electrical Engineering and Computer Science, The University of Newcastle, University Drive, \\ New South Wales 2287, Australia
}

(Received 9 May 2008; accepted 8 June 2008; published online 2 July 2008)

\begin{abstract}
Piezoelectric tube scanners with quartered external electrodes are the most widely used nanopositioning technology in modern scanning probe microscopes. There has been increasing interest in utilizing feedback control techniques to improve bandwidth and accuracy of these nanopositioners. The use of feedback requires a sensor to be incorporated into the nanopositioning device. Noncontact displacement sensors, e.g., capacitive and inductive sensors, have been used for this purpose. However, their measurements contain a significant noise component if operated over large bandwidths. The piezoelectric voltage induced in a tube nanopositioner has been proposed recently as an alternative measure of displacement with a much improved noise figure, up to three orders of magnitude better than capacitive sensors. In this arrangement, an electrode is used to actuate the tube, while the opposite electrode is used as a sensor. This approach has two drawbacks: (i) the operating range of the tube is reduced to half and (ii) the tube is not driven symmetrically, thus the opposite sides of the tube experience asymmetric stresses, i.e., in this mode of operation, the scanner is not a perfectly collocated system. In this paper, we present a new electrode pattern for piezoelectric tube scanners which addresses the above problems and allows simultaneous sensing and actuation of the tube in an efficient way. (C) 2008 American Institute of Physics.
\end{abstract}

[DOI: $10.1063 / 1.2952506$ ]

\section{INTRODUCTION}

The use of a piezoelectric tube actuator with quartered external electrodes for three-dimensional (3D) positioning in a scanning probe microscope (SPM) was first reported in Ref. 1. The majority of commercially available SPMs use a piezoelectric tube scanner for $x-y-z$ positioning. These nanopositioners have a simple construction, are easy to integrate into a microscope, and are relatively inexpensive. The piezoelectric tube scanner reported in Ref. 1 comprises a tube of radially poled piezoelectric material, four external electrodes, and an internal electrode. One end of the tube is fixed and the other end is free to move. Opposite external electrodes are segmented in four equal sectors of $90^{\circ}$. Opposite electrodes are driven by signals of identical magnitude, but opposite signs. Once a voltage is applied to these electrodes, one side of the tube extends vertically, while the opposite side contracts, resulting in bending. This, in turn, results in a two dimensional movement of the free end of the tube in the $x-y$ plane. For $x-y$ actuation, the inner electrode is grounded. To actuate the tube in the $z$ direction, however, the inner electrode is driven by the $z$ signal. Appropriate application of the three signals allows for full $x-y-z$ positioning of the free end of the tube. 3D deflections of a tube with quartered external electrodes, as well as its dynamics are well understood. ${ }^{2-4}$

There has been a consistent effort in recent years to improve accuracy and speed of SPMs using feedback control techniques, see, e.g., Refs. 5-10 and review articles. ${ }^{11,12}$ The key idea associated with feedback-based methods is to damp the first resonant mode of the piezoelectric tube actuator. This "flattening" of the frequency response of the scanner will allow tracking of a faster triangular waveform, and con- sequently a faster scan. Furthermore, in order to achieve accurate positioning at high frequencies, and to minimize the adverse effect of hysteresis, the feedback gain is often chosen to be high. If the tube is driven by a charge source, however, the effect of hysteresis is substantially diminished, ${ }^{13}$ and thus the need for high-gain control can be alleviated.

A major complication with the above feedback-based techniques is their need for a displacement sensor. Capacitive sensors appear to be the most widely used displacement measurement device for such applications. These sensors typically have a rms noise in the order of $20 \mathrm{pm} / \sqrt{\mathrm{Hz}} \cdot{ }^{14,15}$ If the sensor is operated over a bandwidth of $10 \mathrm{kHz}$, this amounts to an rms noise of $2 \mathrm{~nm}$, clearly inadequate for scanning tunneling microscopy, and other applications that require subnanometer precision.

An idea that has emerged very recently ${ }^{13,16}$ is to use a piezoelectric scanner simultaneously for sensing and actuation. In this arrangement one of the quartered electrodes is used to actuate the tube, while the opposite electrode is used as a sensor. In comparison with capacitive sensors, the piezoelectric sensor has a much better noise profile, about three orders of magnitude better. ${ }^{14,15,17}$ This has motivated recent research aimed at using the piezoelectric strain-induced voltage in a feedback loop to augment the tube's damping. This idea was recently implemented in Refs. 18 and 16 with noticeable success. In this approach, the piezoelectric voltage is used as a measurement, and a controller is designed to add substantial damping to the first resonant mode of the tube. This flattens the tube's frequency response and the better damped scanner is then driven by a triangular signal for ras- 


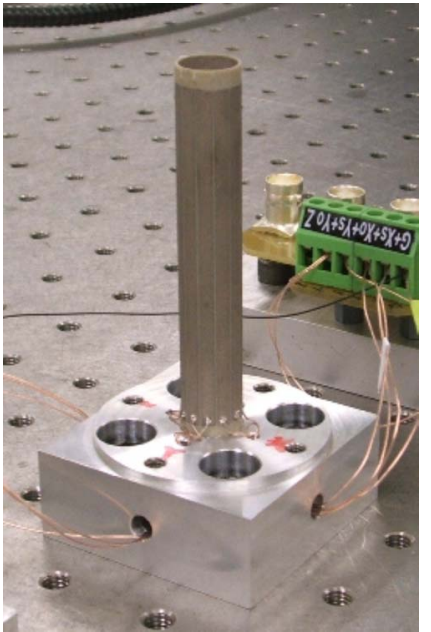

FIG. 1. (Color online) Picture of the experimental apparatus.

tering. Due to its flatter frequency response, the "controlled" scanner can be used at much higher speeds. This, in turn, results in faster scans. The ability to generate and track fast raster scans is necessary for emerging rapid scanning probe microscopy applications. ${ }^{19,20}$

Operating the tube in this mode has two drawbacks: (i) the operating range of the tube is reduced to half since only one electrode is used for actuation and (ii) the tube is driven asymmetrically, thus the opposite sides of the tube are no longer stressed antisymmetrically, i.e., the tube cannot be considered as a perfectly collocated system. The first problem has obvious consequences, while the second complicates the task of designing an efficient controller. These complications arise since the tube with quartered external electrodes is designed to function as an actuator, not as a sensor. In this paper we propose a new electrode pattern that allows both functionalities to be incorporated into the same device in a satisfactory manner.

The remainder of the paper continues as follows. Section II describes the proposed electrode pattern for simultaneous sensing and antisymmetric actuation of a piezoelectric tube scanner. Section III reports the experimental results obtained from the scanner. Properties of the proposed actuator are compared to a regular scanner with quartered external electrodes in this section. Finally Sec. IV concludes the paper.

\section{PIEZOELECTRIC SCANNER WITH A NEW ELECTRODE PATTERN}

A picture of the experimental setup can be viewed in Fig. 1 and schematics of the proposed scanner are shown in Fig. 2. The tube was manufactured by Boston Piezo-Optics. It is made of PZT5A piezoelectric material and is poled radially outward. One end of the tube is mounted on an aluminum holder using strong adhesive material. The setup is bolted to an optical table to minimize the effect of floor vibrations on the measurements obtained from the scanner.

Inside of the tube is covered with a continuous electrode. This electrode is grounded at all times. The outer electrode is segmented into 12 equal sectors of $30^{\circ}$ each. If every three adjacent electrodes are short-circuited together, the tube can

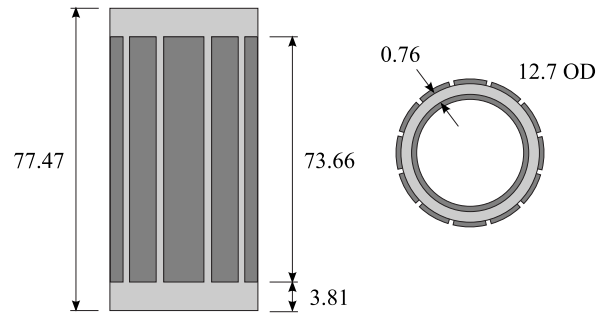

FIG. 2. The proposed piezoelectric tube scanner. Tube dimensions are in millimeters. The tube is manufactured by Boston Piezo-Optics from PZT-5A piezoelectric ceramic material. The outer electrode of the tube is segmented into 12 equal sections.

be used as a regular scanner with quartered external electrodes. This arrangement is illustrated in Fig. 3(a) for simultaneous actuation and sensing in the $x$ direction. The remainder of electrodes are grounded. However, actuation and sensing in the $y$ direction would be identical to $x$.

In this paper we propose the arrangement illustrated in Fig. 3(b). Here, the two outer electrodes on opposite sides are used to actuate the tube. The voltages applied to the opposite electrodes are equal in magnitude, but $180^{\circ}$ out of phase. In this mode of actuation, one side of the tube ex-

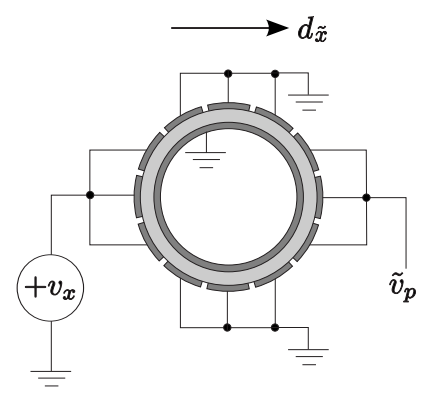

(a)

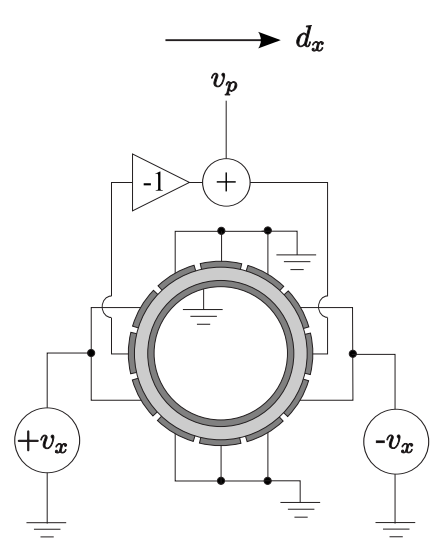

(b)

FIG. 3. The tube is divided into four 90 quadrants. Each quadrant consists of three electrodes. Two sets of experiments were performed on the tube. (a) The tube was wired up as a regular quartered electrode scanner. A voltage $\widetilde{v}_{x}$ was applied to the three electrodes in one quadrant and the strain-induced voltage $\widetilde{v}_{p_{x}}$ was measured at the opposite quadrant electrodes. Displacement $\tilde{d}_{x}$ was also measured at the tip of the tube using a laser vibrometer. (b) A voltage $+v_{x}$ was applied to the two outer electrodes of a quadrant and an equal but opposite voltage $-v_{x}$ was applied to the opposite electrodes. The piezoelectric strain voltage induced at one of the midle electrodes was inverted and added to the voltage measured at the opposite electrode, resulting in the measurement signal $v_{p_{x}}$. Displacement $d_{x}$ was measured at the tip of the tube. 

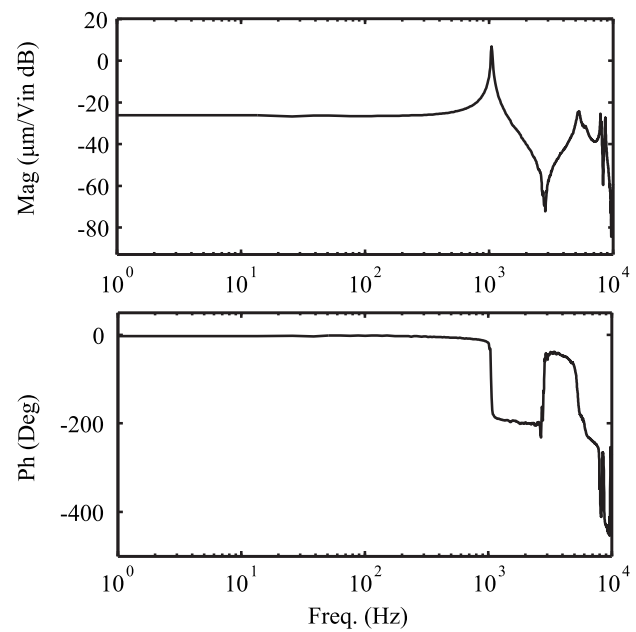

(a)
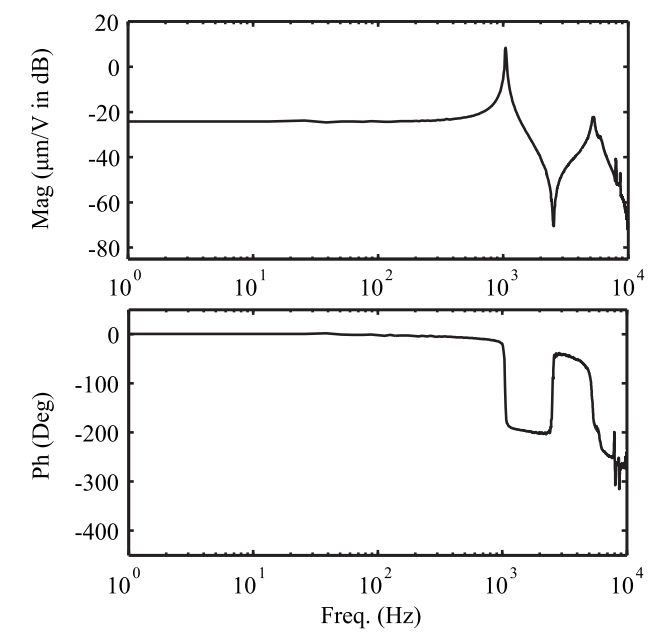

(c)
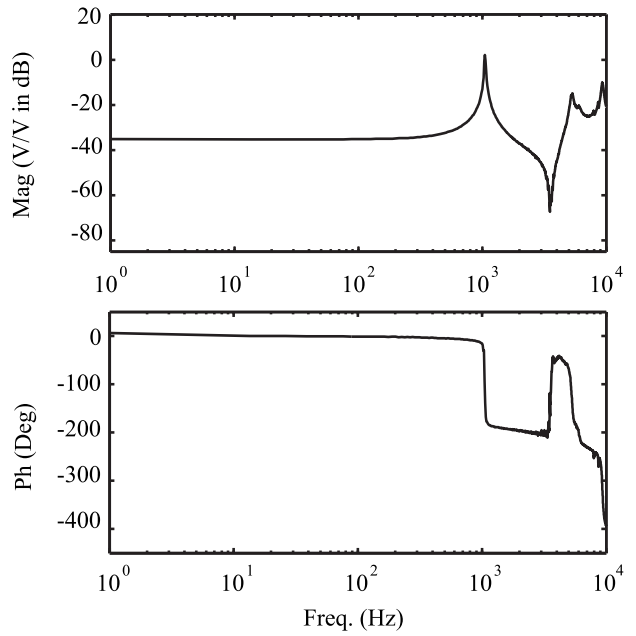

(b)
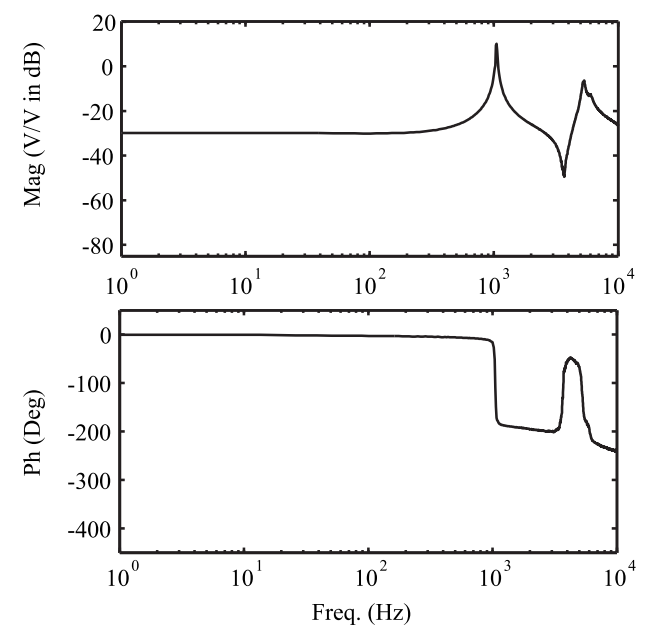

(d)

FIG. 4. Frequency responses of the scanner, [(a) and (b)] when driven asymetrically; [(c) and (d)] under antisymetrtical actuation. (a) and (c) display the displacement response of the tube while (b) and (d) correspond to the voltage measured at the piezoelectric strain sensor.

tends, while the opposite side contracts by the same amount. This results in bending. Furthermore, due to the antisymmetric nature of the actuation, the stress at the opposite sides of the tube will be antisymmetrical. ${ }^{2}$ Due to the piezoelectric effect, ${ }^{21}$ this translates into a voltage at the central electrode of each side. Moreover, due to the antisymmetric nature of the stress, the voltages induced in the opposite electrodes will be of equal amplitude, but $180^{\circ}$ out of phase. As illustrated in Fig. 3(b) the voltage induced at one of the electrodes is inverted and then added to the voltage measured at the opposite electrode.

There are two possibilities to actuate the tube in the $z$ direction. Instead of grounding the inner electrode, a voltage $v_{z}$ can be applied to it. Depending on the polarity of this voltage, the tube will either elongate or shorten in the $z$ direction. The alternative approach requires a continuous circumferential electrode to be deposited on the top exterior of the tube. In the latter approach the inner electrode is at all times grounded, thus the tube is electrically a grounded load.

The arrangement described above allows for simultaneous actuation and sensing with a piezoelectric tube scanner. From a systems point of view, when actuated in one direction, the tube can be considered as a single-input two- output system with the voltage $v_{x}$ as the input and the displacement, $d_{x}$ and the piezoelectric voltage $v_{p_{x}}$ as the output. This SITO system can be described mathematically as

$$
\left[\begin{array}{c}
d_{x} \\
v_{p_{x}}
\end{array}\right]=\left[\begin{array}{c}
G_{d v}^{x}(s) \\
G_{v v}^{x}(s)
\end{array}\right] v_{x} .
$$

The two transfer functions $G_{d v}^{x}(s)$ and $G_{v v}^{x}(s)$ have identical poles. Thus, a controller that augments the damping of $G_{v v}^{x}(s)$ will have a similar effect on $G_{d v}^{x}(s)$. Therefore, the dynamics of the scanner can be significantly improved by establishing a feedback loop that uses $v_{p_{x}}$ as a measurement. This removes the need for incorporating a displacement sensor into scanner apparatus.

When used for simultaneous sensing and actuation the tube with this electrode arrangement has a number of advantages compared to an identical tube with quartered external electrodes. When the tube is operated in the quartered electrode mode, an actuation signal is applied to one of the electrodes and the voltage at the opposite electrode is used for measurement. Let us assume that for the quartered electrode case illustrated in Fig. 3(a) and for a given input signal, $v_{x}$ the tube's deflection is $\tilde{d}_{x}$ and the voltage measured at the 
opposite electrode is $\widetilde{v}_{p_{x}}$. If the same input signal is applied to the tube illustrated in Fig. 3(b), one would expect the free end of the tube to undergo a deflection of about $\frac{4}{3} \widetilde{d}_{x}$. One would also expect to measure a voltage of somewhat less than $2 \widetilde{v}_{p_{x}}$ at the sensing electrode. Hence, with the arrangement proposed here the displacement range of the tube is improved by over $30 \%$, and one would observe an improvement of the signal to noise ratio at the sensing electrodes. It should be pointed out that these predictions are based on a number of simplifying assumptions. However, they turn out to be in agreement with experimental results presented in the next section.

Another important advantage of the proposed scanner is that the tube is now being operated in a fully symmetrical mode. This has important implications for control design which will be discussed in the sequel.

\section{EXPERIMENTAL RESULTS AND DISCUSSION}

A number of experiments were performed to compare properties of the proposed scanner with a regular quartered electrode scanner. In one set of experiments the external electrodes of the tube were wired up as illustrated in Fig. 3(a). This arrangement is almost identical to the case when the tube is being operated as a scanner with quartered external electrodes, where opposite electrodes are used for actuation and sensing. ${ }^{16,18}$ In a second set of experiments the electrodes were wired up as shown in Fig. 3(b). The purpose of the experiments is to actuate the tube in one direction only. If the tube were to be actuated simultaneously in both directions, as is the case in SPMs, the $y$-axis electrodes would have to be utilized in a similar manner to the $x$-axis electrodes.

Capacitance of each electrode was measured using a HIOKI 3532 LCR impedance bridge. It was found that each electrode had a capacitance of approximately $4 \mathrm{nF}$. In both set of experiments, the actuating electrodes were driven by high voltage ACX amplifiers. When driving a capacitive load of $4 \mathrm{nF}$, these amplifiers have a bandwidth of $6.8 \mathrm{kHz}$. This is reflected in the frequency responses obtained from the scanner. The displacement of the free end of the tube was measured using a OFV-552 laser vibrometer. Voltages measured at the sensing electrodes were fed to Stanford Research Systems SR560 low noise preamplifiers. This preamplifier has an input impedance of $100 \mathrm{M} \Omega$. Together with the $4 \mathrm{nF}$ capacitance of the sensing electrode, this pushes the cutoff frequency of the sensing device to well below $1 \mathrm{~Hz}^{22}$ The frequency responses were obtained using a HP-35670A spectrum analyzer. These are plotted in Figs. 4(a)-4(d). Each pair of frequency response functions describes spectral properties of the single-input two-output transfer function matrix in Eq. (1).

There are clear differences between the two pair of frequency response functions. The differences are particularly more observable at higher frequencies. The key reason for these variations is that the quartered electrode tube is actuated asymmetrically, but the 12-electrode tube undergoes antisymmetric actuation. A feature of the proposed scanner which turns out to be important for control applications is
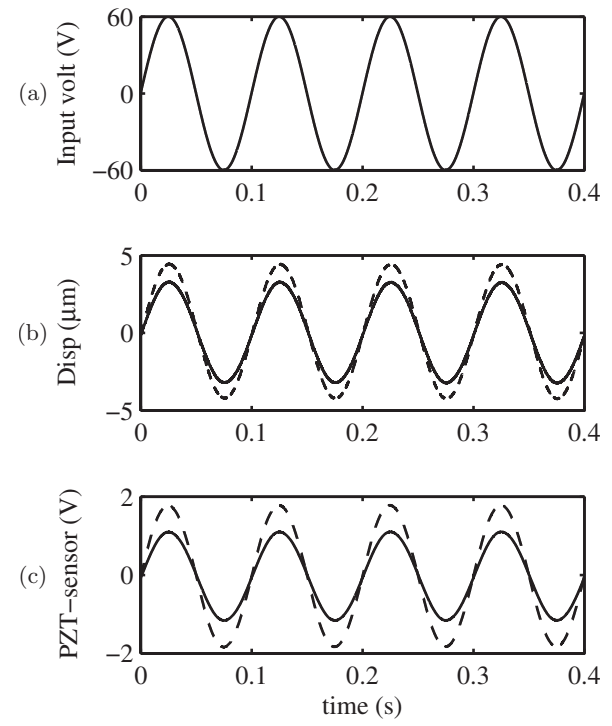

FIG. 5. Measured tip displacements, $x(-)$ and $\tilde{x}(--)$ in $\mu \mathrm{m}$, and piezoelectric strain voltage, $v_{p}(-)$ and $\widetilde{v}_{p}(-)$ in volts.

that its frequency response is much closer to a perfectly collocated system. This would allow one to employ efficient control design tools that are specifically designed for collocated systems, e.g., resonant control, ${ }^{23}$ positive position feedback control, ${ }^{24-26}$ integral resonant control, ${ }^{27}$ and such controllers that may be proposed in the future due to recent progress in understanding stability properties of negative imaginary systems. ${ }^{28}$ Inspection of the FRFs in Figs. 4(a)-4(d) shows that the phase of both displacement and voltage transfer functions appear to roll off at higher frequencies. The phase of a collocated system, however, is expected to remain between 0 and $-180^{\circ} .{ }^{29}$ The apparent rolloff is due to the limited bandwidth of the high voltage amplifier used in the experiments, as articulated above, and can be accounted for in any control design scenario.

Another important advantage of the proposed scanner is that, for the same input signal, it has a larger displacement range and strain-induced voltage when compared to the quartered electrode tube. To verify this, a sinusoidal voltage with amplitude of $60 \mathrm{v}$ and frequency of $10 \mathrm{~Hz}$ was applied to the tube with the electrode arrangements shown in Figs. 3(a) and 3(b). The displacement signals and strain-induced voltages measured at the sensing electrodes are plotted in Fig. 5. For the 12-electrode tube the displacement and the piezoelectric voltage are larger by about $34 \%$ and $61 \%$ compared to similar measurements obtained from the quartered electrode tube. Thus confirming other benefits one could obtain from the proposed scanner.

\section{CONCLUSIONS AND FUTURE WORK}

Piezoelectric tube scanners are the most widely used scanning technology in SPMs. In this paper we proposed a new electrode pattern that allows a scanner to be used, simultaneously, for actuation and sensing. The built-in sensing capability of the scanner turns out to be an important feature of the proposed tube allowing one to address one of the most troublesome properties of the device-the tube's highly resonant nature. By using the strain-induced voltage measured at 
the sensing electrodes, one can augment the damping of the system using a feedback controller. Furthermore, due to the fact that electrodes on opposite sides of the tube are used for actuation, the tube's deflection range is improved and its transfer function resembles that of a collocated system with an alternating pole-zero pattern. The latter has important implications in terms of control design for the tube. Future work involves using feedback control design techniques, specifically tailored for collocated systems, to improve the damping of the tube. This is expected to result in much faster scanners that can be easily incorporated into existing (SPMs).

\section{ACKNOWLEDGMENTS}

This research was supported by the Australian Research Council Centre of Excellence for Dynamic Systems and Control.

${ }^{1}$ G. Binnig and D. P. E. Smith, Rev. Sci. Instrum. 57, 1688 (1986).

${ }^{2}$ C. J. Chen, Ultramicroscopy 42, 1653 (1992).

${ }^{3}$ M. E. Taylor, Rev. Sci. Instrum. 64, 154 (1993).

${ }^{4}$ E. Meyer, H. J. Hug, and R. Bennewitz, Scanning Probe Microscopy (Springer, Heidelberg, Germany, 2004).

${ }^{5}$ R. C. Barrett and C. F. Quate, Rev. Sci. Instrum. 62, 1393 (1991).

${ }^{6} \mathrm{~N}$. Tamer and M. Dahleh, Proceedings of the 33rd IEEE Conference on Decision and Control, Lake Buena Vista, FL, 1994 (unpublished), pp. 1826-1831.

${ }^{7}$ A. Daniele, S. Salapaka, M. V. Salapaka, and M. Dahleh, Proceedings of the American Control Conference, 1999 (unpublished), pp. 253-257.

${ }^{8}$ O. M. El Rifai and K. Youcef-Tomi, Proceedings of the American Control Conference, Arlington, VA, 2001 (unpublished), pp. 3251-3255.

${ }^{9}$ G. Schitter and A. Stemmer, IEEE Trans. Control Syst. Technol. 12, 449 (2004).
${ }^{10}$ G. Schitter, P. Menold, H. F. Knapp, F. Allgower, and A. Stemmer, Rev. Sci. Instrum. 72, 3320 (2001).

${ }^{11}$ S. Devasia, E. Eleftheriou, and S. O. R. Moheimani, IEEE Trans. Control Syst. Technol. 15, 802 (2007).

${ }^{12}$ S. Salapaka and M. Salapaka, IEEE Control Syst. Mag. 28, 65 (2008).

${ }^{13}$ A. J. Fleming and S. O. R. Moheimani, IEEE Trans. Control Syst. Technol. 14, 33 (2006).

${ }^{14}$ A. J. Fleming, A. Wills, and S. O. R. Moheimani, Proceedings of the IEEE/ASME Conference on Advanced Intelligent Mechatronics, Zurich, Switzerland, 2007.

${ }^{15}$ A. J. Fleming, A. Wills, and S. O. R. Moheimani, IEEE Trans. Control Syst. Technol. (unpublished).

${ }^{16}$ B. Bhikkaji, M. Ratnam, A. J. Fleming, and S. O. R. Moheimani, IEEE Trans. Control Syst. Technol. 5, 853 (2007).

${ }^{17}$ I. A. Mahmood, S. O. R. Moheimani, and K. Liu, IEEE Trans. Nanotechnol. (unpublished).

${ }^{18}$ B. Bhikkaji, M. Ratnam, and S. O. R. Moheimani, Sens. Actuators, A 135, 700 (2007).

${ }^{19}$ T. Ando, N. Kodera, D. Maruyama, E. Takai, K. Saito, and A. Toda, Jpn. J. Appl. Phys., Part 1 41, 4851 (2002).

${ }^{20}$ N. Kodera, H. Yamashita, and T. Ando, Rev. Sci. Instrum. 76, 1 (2005).

${ }^{21}$ T. Ikeda, Fundamentals of Piezoelectricity (Oxford University Press, New York, 1996).

${ }^{22}$ T. E. Alberts, T. V. DuBois, and H. R. Pota, Control Eng. Pract. 3, 163 (1995).

${ }^{23}$ S. O. R. Moheimani and B. J. G. Vautier, IEEE Trans. Control Syst. Technol. 13, 1021 (2005).

${ }^{24}$ C. J. Goh and T. K. Caughey, Int. J. Control 41, 787 (1985).

${ }^{25}$ J. L. Fanson and T. K. Caughey, AIAA J. 28, 717 (1990).

${ }^{26}$ S. O. R. Moheimani, B. J. G. Vautier, and B. Bhikkaji, IEEE Trans. Control Syst. Technol. 14, 443 (2006).

${ }^{27}$ S. Aphale, A. J. Fleming, and S. O. R. Moheimani, Smart Mater. Struct. 16, 439 (2007).

${ }^{28}$ A. Lanzon and I. R. Petersen, Proceedings of the 2007 European Control Conference, San Diego, CA, USA, 2006 (unpublished), pp. 31-36.

${ }^{29}$ A. Preumont, Vibration Control of Active Structures (Kluwer, Dordrecht, 1997). 